\title{
Artrodese de coluna: avaliação da satisfação dos cuidadores de pacientes com síndrome de Rett
}

\author{
Arthrodesis spine: satisfaction evaluation of the caregivers of patients \\ with Rett syndrome
}

\author{
Artrodesis de la columna: evaluación de la satisfacción de los \\ cuidadores de pacientes con síndrome de Rett
}

\author{
Cleverson Tadeu Sidoli ${ }^{1}$ \\ Edson Pudles ${ }^{2}$ \\ Luís Eduardo Munhoz da Rocha ${ }^{3}$ \\ Luiz Antônio Munhoz da Cunha ${ }^{4}$
}

\section{RESUMO}

Objetivo: avaliar o nível de satisfação dos cuidadores de pacientes com síndrome de Rett. Métodos: foi realizado um estudo retrospectivo por meio dos prontuários de dez pacientes com Síndrome de Rett, nove femininos e um masculino. Foram obtidos os dados radiográficos e foi elaborado um questionário de 31 perguntas, respondidas pelos familiares dos pacientes. Resultados: a redução média no ângulo pré-operatório e a medida no pós-imediato foi de $72,4 \%$. Complicação pós-operatória ocorreu em apenas um caso, e 87,5\% dos cuidadores afirmaram que houve melhora expressiva com relação ao efeito da cirurgia sobre os cuidados pessoais, na função e locomoção; o tempo de permanência na cadeira de rodas aumentou. Para $66,7 \%$ dos cuidadores, a frequência de internamentos por problemas médicos e a frequência de pneumonia diminuíram com a cirurgia. Também fizeram uma avaliação positiva sobre a estética da deformi-

\section{ABSTRACT}

Objective: to evaluate the satisfaction of the caregivers of Rett Syndrome (RS) patients. Methods: For this retrospective study, statistical data were presented by the medical records of 10 patients with $R S$, nine females and only one male. With the radiological data presented in the medical records, a questionnaire was prepared with 31 questions to be answered by family. Results: the average reduction of the angle that occurred between the preoperative evaluation and the postoperative evaluation was of $72.4 \%$. Postoperative complications occurred in only one case, and $87.5 \%$ of the caregivers said that there was significant improvement with respect to the effect of surgery on personal care, function, locomotion; the time in the wheelchair increased. To $66.7 \%$ of the caregivers, with the surgery, the frequency of medical admissions and the frequency of pneumonia decreased. They also made a positive evaluation about the aesthetic deformity of the spine. The vast majority of the caregivers

\section{RESUMEN}

Objetivo: evaluar el nivel de satisfacción de los cuidadores de pacientes con sindrome de Rett. Métodos: fue realizado un estudio retrospectivo por medio de las historias clinicas de 10 pacientes con sindrome de Rett, nueve mujeres y un hombre. Fueron obtenidos datos radiográficos y se elaboró un cuestionario de 31 preguntas a los familiares de los pacientes. Resultados: la reducción promedio en el ángulo preoperatorio y el promedio en el postoperatorio fue del 72,4\%. La complicación postoperatoria ocurrió en solo un caso y el $87,5 \%$ de los cuidadores afirmaron que hubo mejoría expresiva con relación al efecto de la cirugía sobre los cuidados personales, en la función y locomoción, y además, el tiempo de permanencia en la silla de ruedas aumentó. Para el 66,7\%, de los cuidadores, la frecuencia de internamientos por problemas médicos y la frecuencia de neumonía disminuyeron con la cirugía. También hicieron una evaluación positiva sobre la estética de la deformidad de la columna. La gran

\footnotetext{
Trabalho realizado no Hospital Infantil Pequeno Príncipe - Curitiba (PR), Brasil.

'Médico especializando em Coluna (2009) pelo Hospital Universitário Evangélico de Curitiba - Curitiba (PR), Brasil.

2Preceptor da Residência Médica do Hospital Universitário Evangélico de Curitiba - Curitiba (PR), Brasil.

${ }^{3}$ Preceptor da Residência Médica do Hospital Pequeno Príncipe - Curitiba (PR), Brasil.

4Professor Titular da Disciplina de Ortopedia e Traumatologia da Universidade Federal do Paraná - UFPR - Curitiba (PR), Brasil; Médico do Serviço de Ortopedia do Hospital Pequeno Príncipe - Curitiba (PR), Brasil.
} 
dade da coluna. A grande maioria dos cuidadores $(85,7 \%)$ submeteria novamente seus filhos à cirurgia e recomendariam para outra criança com o mesmo problema. Conclusão: houve redução significativa na média do ângulo de Cobb entre o pré e pós-operatório e melhoria em todos os aspectos do paciente. Os cuidadores manifestaram que submeteriam seus filhos ao procedimento cirúrgico novamente.
(87.5\%) said that their children could undergo surgery again and they would recommend it to another child with the same problem. CONCLUSION: there was a significant reduction in the mean Cobb anglebetween the preoperative and postoperative period, an improvement in all aspects of the patient, and caregivers also expressed that their children could undergo to surgery again. mayoría de los cuidadores (el 85,7\%) sometería nuevamente sus hijos a la cirugía y recomendaría a otro niño con el mismo problema hacer este tipo de corrección. Conclusión: hubo una reducción significativa en el promedio del ángulo de Cobb entre el pre y postoperatorio y una mejoría en todos los aspectos del paciente. Los cuidadores manifestaron que someterían sus hijos de nuevo al procedimiento quirúrgico.

\author{
DESCRIPTORES: Síndrome de \\ Rett/cirugía; Escoliosis; \\ Fusión vertebral; Cuidadores
}

cirurgia; Escoliose; Fusão vertebral; Cuidadores
KEYWORDS: Rett syndrome/ surgery; Scoliosis; Spinal fusion; Caregivers

\section{INTRODUÇÃO}

A Síndrome de Rett (SR) é uma desordem neurológica de causa genética descoberta em 1988 e esporádicas envolvendo as mutações do gene conhecido como MecP21,2. Com incidência de um caso para cada 10 mil a 15 mil meninas nascidas vivas, a SR, a priori, afeta apenas crianças do sexo feminino que, com nascimento e desenvolvimento aparentemente normais até os 6 a 18 meses, começam, a partir de então, a demonstrar os sinais clínicos da condição, que evolui em quatro estágios progressivos, mas não degenerativos. Os sinais clínicos são: estagnação do desenvolvimento, desaceleração do crescimento do perímetro cardíaco e do ganho ponderal, hipotonia, regressão de aquisições já adquiridas (fala ou marcha, por exemplo), alterações comportamentais (choro intenso aparentemente desmotivado e crise de irritabilidades longas, autoagressão), desvios no contato social e na comunicação, isolamento (características ditas autísticas), perda gradual do uso prático das mãos e da linguagem, aparecimento de movimentos manuais estereotipados (sinal muito característico da SR), aparecimento de crises convulsivas (em 50\% a 70\% dos casos), alterações respiratórias importantes (apneia, hiperventilação, perda de fôlego, aerofagia), alterações de sono (insônia), bruxismo intenso e constipação intestinal.

Em torno dos dez anos de idade, embora os sintomas clínicos acima descritos tendam a melhorar, há lenta deterioração motora progressiva, resultando em escoliose grave, distúrbios vasomotores e atrofia muscular. Muitas das que ainda andam podem perder a marcha nesta etapa da vida.

Em termos cognitivos, permanecem, ao que se sabe, em estágio correspondente ao desenvolvimento intelectual dos 12 aos 24 meses. A linguagem está sempre ausente. Trata-se, portanto, de condição que resulta em múltiplas deficiências e, portanto, na total dependência da pessoa por ela acometida, que deverá estar sob cuidado de familiares e/ou responsáveis 24 horas por dia.

\section{MÉTODOS}

Análise retrospectiva de prontuários de dez pacientes com $\mathrm{SR}$, sendo que nove destes eram do sexo feminino e apenas um do sexo masculino.

Após análise das radiografias pré e pós-operatórias para mensuração dos ângulos de Cobb, também foram analisadas as radiografias do quadril desses pacientes quanto à centralização da articulação coxofemoral. Também foram analisadas a obliquidade pélvica e a técnica operatória utilizada (via anterior, via posterior ou ambas), com os seus respectivos níveis de instrumentação.

Após coleta de dados (idade, sexo, idade no momento cirúrgico), os pacientes foram tomados para reavaliação ambulatorial e, neste momento, seus familiares responderam a um questionário elaborado com 31 perguntas referentes aos cuidados pessoais; função e locomoção; estado geral de saúde; e estética.

Os resultados obtidos no estudo foram expressos por médias e desvios-padrão ou por frequências e percentuais.

\section{RESULTADOS}

Neste estudo, foram incluídos dez pacientes portadores de Síndrome de Rett submetidos à artrodese da coluna. Nove pacientes eram do gênero feminino e apenas um do gênero masculino. A média de idade no início do tratamento foi de $11,8 \pm 3,5$ anos e, no momento da cirurgia, foi de $14,2 \pm$ 2,4 anos, sendo o intervalo médio decorrido entre o início do tratamento e a cirurgia igual a 2,4 $\pm 3,0$ anos.

A classificação I na Fundação Mundial de Saúde (FMS) foi observada em cinco pacientes, a classificação II em dois deles e três pacientes tinham a classificação V. A avaliação do quadril indicou que cinco pacientes tinham quadril centrado, outros quatro, subluxado, e apenas um luxado. A redução média no ângulo ocorrida entre a avaliação pré-operatória e a avaliação no pós-imediato foi de $72,4 \%$, diminuindo discretamente na avaliação da última consulta, quando a média passou para $71 \%$. Os limites da curva e os ângulos medidos 
nas avaliações pré-operatórias, no pós-imediato e na última consulta a partir de radiografias são apresentados na Tabela 1 .

Os pacientes foram avaliados por seus cuidadores quanto aos efeitos da cirurgia a que foram submetidos. $\mathrm{Na}$ abordagem, foram consideradas questões relacionadas aos cuidados pessoais, função e locomoção, estado geral de saúde, estética, estado emocional e recomendações sobre a cirurgia.

Complicação pós-operatória ocorreu em apenas um caso. Quanto aos cuidados pessoais, na opinião de pelo menos $83 \%$ dos cuidadores houve uma grande melhora em atividades como vestir ou tirar as calças, alimentar e tomar banho. A troca de fraldas e a higiene da área genital, para aproximadamente metade dos cuidadores, foram itens que melhoraram pouco ou não apresentaram alterações. $\mathrm{Na}$ avaliação do efeito da cirurgia sobre os cuidados pessoais em geral, 87,5\% dos cuidadores afirmaram que houve melhora expressiva nessas atividades (Tabela 2).

Nas questões sobre função e locomoção, considerando-se a avaliação da maioria dos cuidadores, o efeito positivo da cirurgia foi observado em atividades como colocar ou tirar a criança da cadeira de rodas, sentar na cadeira de rodas, deitar ou levantar da cama e a posição na cama. Já o tempo de permanência na cadeira de rodas e em uma cadeira comum piorou após a cirurgia (Tabela 3 ).
Para $66,7 \%$ dos cuidadores, a frequência de internamentos por problemas médicos e pneumonia diminuíram com a cirurgia. A frequência de infecção urinária, na avaliação de quatro de seis cuidadores, não se alterou. Nenhum item sobre o estado geral de saúde foi relatado como tendo aumentado em frequência após a cirurgia (Figura 1).

Uma avaliação positiva sobre a estética da deformidade da coluna foi feita por todos os cuidadores. Para 14,3\% deles houve um pouco de melhora, e para $85,7 \%$ houve muita melhora.

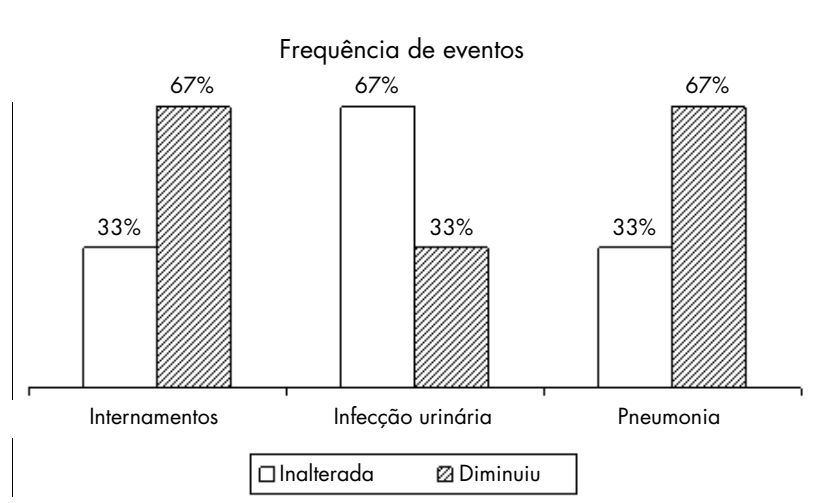

Figura 1

Avaliação da frequência de internamentos por problemas médicos, de infecção urinária e de pneumonia

\section{TABELA 1 - Limites da curva e ângulos no pré-operatório, pós-imediato e na última consulta}

\begin{tabular}{lcccccc}
\hline \multirow{2}{*}{ Paciente } & $\begin{array}{c}\text { Limites da } \\
\text { curva }\end{array}$ & \multicolumn{3}{c}{ Ângulo (em graus) } & \multicolumn{2}{c}{ Redução \% do ângulo } \\
\cline { 3 - 7 } & Pré-operatório & Pós-imediato & $\begin{array}{c}\text { Última } \\
\text { consulta }\end{array}$ & Pré-pós imediato (\%) & Pré-última consulta (\%) \\
\hline 1 & T2-L5 & 90 & 33 & 30 & 63,3 & 66,7 \\
2 & T2-L3 & 50 & 13 & & 74 & 74,4 \\
3 & T2-L4 & 78 & 18 & 20 & 76,9 & 65,2 \\
4 & T4-L5 & 46 & 15 & 16 & 67,4 & 79,5 \\
5 & T10-L5 & 39 & 7 & 8 & 82,1 & 76,3 \\
6 & T2-L3 & 80 & 16 & 19 & 80 & 70 \\
7 & T3-L3 & & & & & 73,3 \\
8 & T1-L3 & 30 & 8 & 9 & 62,5 & 71 \\
9 & T1 1-L4 & 40 & 15 & 14 & 72,4 & 5,8 \\
Média & & 56,6 & 14,7 & 18,3 & 7,4 & \\
Desvio-padrão & & 22,6 & 8 & 8,4 &
\end{tabular}

TABELA 2 - Avaliação quanto a atividades relacionadas aos cuidados pessoais

\begin{tabular}{|c|c|c|c|c|c|}
\hline \multirow{2}{*}{ Cuidado pessoal } & \multirow{2}{*}{$\mathrm{n}^{*}$} & Piorou pouco & Sem alteração & \multirow{2}{*}{$\begin{array}{l}\text { Melhorou } \\
\text { pouco }\end{array}$} & \multirow{2}{*}{$\begin{array}{c}\text { Melhorou muito } \\
\mathrm{n}(\%)\end{array}$} \\
\hline & & $\mathrm{n}(\%)$ & $\mathrm{n}(\%)$ & & \\
\hline Vestir/tirar as calças & 7 & & $1(14,3)$ & & $6(85,7)$ \\
\hline Alimentar & 6 & & $1(16,7)$ & & $5(83,3)$ \\
\hline Tomar banho & 8 & & $2(25,0)$ & & $6(75,0)$ \\
\hline Trocar as fraldas & 6 & & $2(28,6)$ & $1(14,3)$ & $4(57,1)$ \\
\hline Realizar higiene da área genital & 7 & & $3(42,9)$ & $1(14,3)$ & $3(42,9)$ \\
\hline Em geral & 8 & $1(12,5)$ & & & $7(87,5)$ \\
\hline
\end{tabular}

* Número de casos válidos. 
TABELA 3 - Avaliação quanto à função e à locomoção

\begin{tabular}{|c|c|c|c|c|c|c|}
\hline \multirow[t]{2}{*}{ Função e locomoção } & \multirow[t]{2}{*}{$\mathrm{n}^{*}$} & \multirow{2}{*}{$\begin{array}{l}\text { Piorou } \\
\text { muito }\end{array}$} & \multirow{2}{*}{$\begin{array}{l}\begin{array}{l}\text { Piorou } \\
\text { pouco }\end{array} \\
\mathrm{n}(\%) \\
\end{array}$} & \multirow{2}{*}{$\begin{array}{c}\begin{array}{c}\text { Sem } \\
\text { alteração }\end{array} \\
\mathrm{n}(\%)\end{array}$} & \multirow{2}{*}{$\begin{array}{c}\begin{array}{c}\text { Melhorou } \\
\text { pouco }\end{array} \\
\mathrm{n}(\%)\end{array}$} & \multirow{2}{*}{$\begin{array}{c}\begin{array}{c}\text { Melhorou } \\
\text { muito }\end{array} \\
\mathrm{n}(\%) \\
\end{array}$} \\
\hline & & & & & & \\
\hline Colocar/tirar a criança da cadeira de rodas & 7 & & & $1(14,3)$ & & $6(85,7)$ \\
\hline Sentar na cadeira de rodas & 7 & & & $1(14,3)$ & & $6(85,7)$ \\
\hline Deitar ou levantar da cama & 8 & & & $3(37,5)$ & & $5(62,5)$ \\
\hline Posição para dormir & 8 & & & $3(37,5)$ & & $5(62,5)$ \\
\hline Sentar em uma cadeira comum & 3 & & $1(33,3)$ & & & $2(66,7)$ \\
\hline Tirar/colocar no carro & 6 & & $2(25)$ & $2(25)$ & & $4(50)$ \\
\hline Levar a cça na cadeira de rodas para outro lugar & 5 & & $1(20)$ & $2(40)$ & & $2(40)$ \\
\hline Colocar as talas e aparelho & 5 & & & $4(80)$ & $1(20)$ & \\
\hline Uso das mãos & 6 & & & $6(100)$ & & \\
\hline Tempo de perman. sentada numa cadeira comum & 6 & $1(16,7)$ & $3(50)$ & $2(33,3)$ & & \\
\hline Tempo de permanência na cadeira de rodas & 8 & $4(57,1)$ & $2(28,6)$ & $1(14,3)$ & & \\
\hline Em geral & 7 & $1(14,3)$ & $1(14,3)$ & & & $5(71.4)$ \\
\hline
\end{tabular}

*Número de casos válidos.

A grande maioria dos cuidadores $(85,7 \%)$ submeteria novamente seus filhos à cirurgia e a recomendariam para outra criança com o mesmo problema. Os mesmos cuidadores que recomendariam a cirurgia relataram que seus filhos estão mais alegres.

\section{DISCUSSÃO}

A escoliose em SR é uma complicação comum como nos outros pacientes com escoliose neuromuscular. $\mathrm{Na}$ $\mathrm{SR}$, a curva progride mais aceleradamente que na coliose idiopática, em torno de 14 a $21^{\circ} \mathrm{em}$ um ano em pequenas séries ${ }^{3-5}$. As evidências para o manejo em escoliose na Síndrome de Rett são limitadas. Alguns casos são englobados nas escolioses neuromusculares, sendo que não há comparação entre os grupos ${ }^{6-8}$. O planejamento cirúrgico deve começar com um ângulo de Cobb entre 40 e $60^{\circ} 7,9$.

O tratamento cirúrgico é indicado para reduzir os efeitos da escoliose e proporcionar uma melhora na qualidade de vida, que traz benefícios para o paciente, como equilíbrio para sentar, e para o manuseio pelos seus cuidadores. Porém, o tratamento cirúrgico é uma decisão difícil para os familiares devido às complicações inerentes ao tratamento 5 . No nosso estudo, tivemos apenas uma complicação pós-operatória, em que foi necessária uma revisão cirúrgica com significativa melhora do quadro.

Em seu estudo, Askin et al. revelam que o decréscimo das funções orgânicas ocorre aproximadamente 12 meses após o aparecimento da escoliose ${ }^{10}$. Algumas das questões abordadas neste estudo mostraram que o tempo de permanência sentado em uma cadeira piorou. Colvin et al. relatam não haver perda das funções pré-existentes com o tratamento cirúrgico da escoliose, além de terem mensurado várias funções da vida diária em seu estudo ${ }^{11}$.

Não foi encontrado estudo semelhante na literatura revisada. Os estudos já revisados foram embasados em grupos populacionais europeus e norte-americanos, portanto, não refletem a nossa realidade.

\section{CONCLUSÃO}

No presente estudo tivemos uma redução significativa na média do ângulo de Cobb entre o pré e o pós-operatório.

Houve melhora em todos os aspectos e, em especial, a maioria dos cuidadores submeteria seus filhos novamente ao procedimento cirúrgico. Os mesmos relatam que seus filhos estão mais alegres.

\section{REFERÊNCIAS}

1. Diagnostic criteria for Rett Syndrome. The Rett Syndrome Diagnostic Criterea Work Group. Ann Neurol. 1988;23(4):425-8.

2. Amir RE, Fang P, Yu Z, Glaze DG, Percy AK, Zoghbi HY, et al. Mutations in exon 1 of MECP2 are a rare cause of Rett syndrome. J Med Genet. 2005;42(2):e15.
3. Keret D, Bassett GS, Bunnell WP, Marks HG. Scoliosis in Rett syndrome. J Pediatr Orthop. 1988;8(2):138-42.

4. Lidström J, Stokland E, Hagberg B. Scoliosis in Rett syndrome. Clinical and biological aspects. Spine (Phila Pa 1976). 1994;19(14):1632-5.

5. Harrison DJ, Webb PJ. Scoliosis in the Rett syndrome: natural history and treatment. Brain Dev. 1990;12(1):154-6.
6. Berven S, Bradford DS

Neuromuscular scoliosis: causes of deformity and principles for evaluation and management. Semin Neurol. 2002;22(2):167-78.

7. Miller F. Spinal deformity secondary to impaired neurologic control. J Bone Joint Surg Am. 2007;89(Suppl 1):1437.

8. Mehta JS, Gibson MJ. The treatment of neuromuscular scoliosis. Curr Orthop 2003;17(4):313-21. 
9. Kerr AM, Webb P, Prescott RJ, Milne Y. Results of surgery for scoliosis in Rett Syndrome. J Child Neurol. 2003;18(10):703-8.

10.Askin GN, Hallett R, Hare N, Webb JK. The outcome of scoliosis surgery in the severely physically handicapped child. An objective and subjective assessment. Spine (Phila Pa 1976). 1997;22(1):44-50.
11. Colvin L, Fyfe S, Leonard S, Schiavello T, Ellaway C, De Klerk N, et al. Describing the phenotype in Rett syndrome using a population database. Arch Dis Child. 2003;88(1):38-43.

\section{Correspondência:}

Cleverson Tadeu Sidoli

Hospital Regional do Litoral

Rua dos Expedicionários, 269

Bairro Palmital

CEP 83206-450

E-mail: cleversonsidoli@hotmail.com 